\title{
$\angle S$ Research Square \\ Challenges in Oral Health Provision and Utilization in the Australian Indigenous Population: A Scoping Review
}

\section{Peivand Bastani ( $\sim$ bastani@sums.ac.ir)}

Shiraz University of Medical Sciences https://orcid.org/0000-0002-0412-0267

\section{Yaser Sarikhani}

Shiraz University of Medical Sciences

\section{Arash Ghanbarzadegan}

The University of Adelaide

\section{Faeze Ostovar}

Shiraz University of Medical Sciences

\section{Lisa Jamieson}

The University of Adelaide

\section{Research}

Keywords: Utilization, Provision, Oral health, Dental health, Indigenous Australian

Posted Date: July 16th, 2020

DOl: https://doi.org/10.21203/rs.3.rs-42699/v1

License: (9) This work is licensed under a Creative Commons Attribution 4.0 International License. Read Full License 


\section{Abstract \\ Background}

Oral health is a vital area of public health. Access to dental services are particularly important among Indigenous populations at a global level. This scoping review aimed to investigate challenges in the provision and utilization of oral health services among Indigenous Australians. The findings may be an important contribution to the literature and facilitate policymakers in reducing inequities in dental service access and utilisation among this vulnerable population.

\section{Methods}

This scoping review was conducted up to June 10,2020. Five main databases were searched, including PUBMED, SCOPUS, ISI Web of Science, EMBASE and PROQUEST. The main key words were: oral health, dental health, utilization, provision, access, Indigenous, Native, Aboriginal and Torres Strait Islander. Arksey and O'Malley`s protocol was applied, which comprises 5 steps of: (1) identifying the research question; (2) identifying relevant studies; (3) study selection; (4) charting the data and collating and; (5) summarising and reporting results. Thematic analysis was used to summarize and analyse the data applying MAX QDA 10 software for qualitative data analysis. For a better understanding of the themes and subthemes, a thematic network was presented.

\section{Results}

Results show that there are six main themes and fourteen sub-themes affecting Indigenous Australians' utilization and provision of oral health services. Personal characteristics, Socio-economic status and challenges regarding access were that the main factors influencing utilization of oral health services. The findings suggest that challenges relating to public health, policymaking and service provision can affect Indigenous Australians provision to oral health services.

\section{Conclusion}

Benefits in Indigenous Australian access and utilization of oral health services may improve if policy makers at both national and local levels increase resourcing in this important public health sector. A deeper understanding of the social and cultural nuances driving Indigenous Australians' reluctance to attend for dental care is crucial.

\section{Introduction}

Oral health is an integral component of public health. Optimal oral health leads to an increase in quality of life and general health [1]. Limitations in dental public health systems, however, can lead to insufficient 
or oral health [2]. Various studies have focused on the effects of these challenges on vulnerable groups and minorities [3, 4].

One of the most vulnerable groups in Australia is the Indigenous community, which comprises approximately $3 \%$ of Australia's total population $[5,6]$. Indigenous Australians have much poorer oral health, irrespective of indictor used, than their non-Indigenous counterparts [7]. For example, Indigenous Australians have a higher rate of dental caries, more untreated teeth, more periodontal problems, lower levels of oral health literacy and lower reported quality of life compared to other people in the community [7-9].

Various challenges have been posed in providing dental services to Indigenous Australians. These include fears of judgement, geographical access, higher costs of preventive and curative services, long waiting times and poor oral health literacy $[10,11]$. There is some evidence that an under-utilisation of dental services can worsen and intensify the oral health conditions of Indigenous Australians [12].

One of the key goals of the Australian Government is to provide adequate access to dental services and to reduce oral health inequality among Indigenous and non-Indigenous Australians [13]. Indigenous Australians (those identifying as Aboriginal and/or Torres Strait Islander) are prioritized in the oral health plans at both a national and state-level $[14,15]$. However, access and utilisation of dental public health services among Indigenous Australians remains low $[7,16,17]$. This has many consequences, with dental problems leading to emotional, physical and financial impacts [12], as well as feelings of shame about personal appearance and self-esteem [8].

Given the lack of empirical evidence on the challenges in the utilisation and provision of oral health services for Indigenous Australians, it became apparent that a scoping study was needed. Therefore, this scoping review aimed to investigate the challenges in oral health provision and utilization among Indigenous Australians to better enable policymakers to make equitable and fair choices regarding funding for oral health services.

\section{Method}

This scoping review was conducted up to June 10,2020. We implemented content analysis and thematic network answer the question: "what are the main challenges in provision and utilization of the oral health services among Indigenous Australians. The scoping review instrument developed by Arksey and O'Malley [18] was applied to construct a comprehensive map of evidence on the research question [19]. The following 6 steps were thus used [18]:

- Identifying the research question

- Identifying relevant studies

- Study selection

- Charting the data 
- Collating, summarising and reporting the results

- Consultation exercise (optional)

\section{Identifying the research question}

The research question of a scoping review determines the scope of the study and provides the parameters for the initial search. At the same time, a scoping review question should include the Population, Content and Context (PPC) of the study [18].

In this study, the population comprised Indigenous Australians. The content was the challenges and barriers in the provision and utilization of oral health services. The context was the historical, social, cultural and political nuances that have shaped Indigenous Australian health and wellbeing for the last 250 years.

The research question for this study was finalized as: "what are the main challenges of oral health provision and utilization among Indigenous Australians"?

\section{Identifying the relevant studies}

Five scientific databases were searched systematically from January 1,2000 , to June 10,2020 . These included PUBMED, SCOPUS, PROQUEST, EMBASE and Web of Science. The search was conducted by applying the following keywords: oral health, dental health, oral care, oral hygiene and delivery, provision, providing, utilization, use, access and challenges, problems, barriers, obstacles, indigence, native, aboriginal, original Australian, Torres Strait Islander and, Australia. The logical operator OR was used between the synonymous keywords, and the logical operator AND was applied to combine them. Table 1 shows details of the search strategy of the scoping review.

\section{Study selection}

Using the above search strategy, 187 articles were selected. This was reduced to 151 after exclusion of duplicates. The manuscripts were first scanned by their titles and abstracts by two of the researchers separately (YS and FO). The selected abstracts were saved in libraries applying EndNote X7.1, by Thomson Reuters. A PRISMA flowchart summarizes this process (Fig. 1). The full texts were then studied individually. In the event of a disagreement (about 2 of the articles), a third researcher (AG) read the paper to achieve consensus. Inclusion criteria included papers with full English text, qualitative or quantitative designs, original research articles or reviews were included. Exclusion criteria included only abstracts in English, letters, commentaries and editorials.

\section{Charting the data}

A data extraction form was applied according to the aim of the study. This form contained the author's name, study title, study topic, study design, year and place of publication and key determinants of specialty selection. After all the included articles were extracted, a content-analysis approach was applied. 


\section{Collating, summarising and reporting the results}

Thematic analysis was used to summarize and categorize the data. Thematic analysis is an inductive approach for content analysis in order to make themes from the data content [20]. Analysis was conducted via six steps as follows: In the first step, familiarization with data occurs through reading of the content several times. Then the initial codes were identified based on the research question and study aim. After reviewing these preliminary codes, the final codes were created. The final codes were merged and categorized to create sub-themes. In the fifth step, the main themes were refined and labelled. Finally, for a better understanding of the themes and subthemes, the results were both tabulated and a thematic network was presented applying MAX QDA 10 software for qualitative data analysis.

Table 1

Search strategy of the scoping review

PUBMED $\quad((((" O r a l$ health" [Title/Abstract] OR "Dental Health" [Title/Abstract] OR "oral hygiene" [Title/Abstract] OR "oral care" [Title/Abstract] OR dental[Title/Abstract])) AND (delivery TTitle/Abstract OR provision [Title/Abstract] OR providing [Title/Abstract] OR utilization TTitle/Abstract OR use [Title/Abstract] OR usage [Title/Abstract] OR access [Title/Abstract] OR accessibility[Title/Abstract])) AND (challenges [Title/Abstract] OR problems [Title/Abstract] OR shortcomings [Title/Abstract] OR barriers [Title/Abstract] OR deficits [Title/Abstract] OR obstacles[Title/Abstract])) AND (Indigenous[Title/Abstract] OR Native[Title/Abstract] OR Aboriginal[Title/Abstract] OR original[Title/Abstract])) OR Torres Strait Islander[Title/Abstract])) AND (Australian[Title/Abstract] OR Australia[Title/Abstract])

SCOPUS ( TITLE-ABS-KEY ("Oral health" OR "Dental Health" OR "oral hygiene" OR "oral care" OR dental) AND TITLE-ABS-KEY (delivery OR provision OR providing OR utilization OR use OR usage OR access OR accessibility) AND TITLE-ABSKEY (challenges OR problems OR shortcomings OR barriers OR deficits OR obstacles) AND TITLE-ABS-KEY (indigenous OR native OR aboriginal OR original OR Torres Strait Islander) AND TITLE-ABS-KEY (Australian OR Australia) )

EMBASE TITLE, ABSTRACT, KEYWORDS: ('oral health' OR 'dental health' OR 'oral hygiene' OR 'oral care' OR dental AND (delivery OR provision OR providing OR utilization OR use OR usage OR access OR accessibility) AND (challenges OR problems OR shortcomings OR barriers OR deficits OR obstacles) AND (indigenous OR native OR aboriginal OR original OR Torres Strait Islander) AND (Australian OR Australia)

PROQUEST TITLE: ("Oral health" OR "Dental Health" OR "oral hygiene" OR "oral care" OR dental OR oral) AND (delivery OR provision OR providing OR utilization OR use OR usage OR access OR accessibility) AND ab(challenges OR problems OR shortcomings OR barriers OR deficits OR obstacles) AND ab(Indigenous OR Native OR Aboriginal OR original OR Torres Strait Islander AND ab(Australian OR Australia)

Web of

Science

TITLE: ("Oral health" OR "Dental Health" OR "oral hygiene" OR "oral care" OR dental) AND ALL FIELDS: (delivery OR provision OR providing OR utilization OR use OR usage OR access OR accessibility) AND ALL FIELDS: (challenges OR problems OR shortcomings OR barriers OR deficits OR obstacles) AND ALL FIELDS: (Indigenous OR Native OR Aboriginal OR original) OR Torres Strait Islander AND ALL FIELDS:

(Australian OR Australia) 
As indicated in Fig. 1, of the 187 initially included articles, 24 were selected for the scoping review. All 24 manuscripts except one was published between 2007 and 2020. Thirteen articles (54.2\%) involved quantitative data and six (25\%) followed a qualitative approach. One study $(4.1 \%)$ adopted a mix method approach, with the remaining four manuscripts being review articles or discussion papers $(16.7 \%)$ (Table 2 Appendix).

There were six main themes and fourteen-related sub-themes identified that indicated the challenges of oral health provision and utilization in the Indigenous Australian context (Table 3). 
Table 3

Challenges of provision and utilization of oral health services among Australian indigenous population

Main themes Sub-themes Final codes

Personal characteristics
Age

Age

\section{Older ages}

The teenage years

Early childhood

Sex Being female

Physiological status

Pregnancy

Perceived severity and pain of oral disease

Dental history and previous dental health status

Psychological status

High levels of psychological distress

Low self-efficacy

Fear

economic

status
Health literacy status

Economic

challenges

Parents` limited oral health knowledge

Low health literacy and wrong perceptions

lack of information

Brushing education utilizing fluoride toothpaste.

Oral health awareness

Financial concerns and lack of enabling resources

Transport problems

Worrying about the future

Juggling multiple priorities

Living in a rural locations

Poor status of oral health and high treatment needs in rural and remote communities

Social and cultural challenges
Urban Indigenous families

Poorer oral health status of oral health of rural dwellers than that of urban dwellers

Not having a culturally appropriate, acceptable and safe environment.

Availability and access to culturally appropriate services 
Final codes

Only visiting a dentist because of a problem (pain existed, emergency services)

Language barriers

Difficulty communicating with clients

Public health

challenges
Public health

behaviours

Health beliefs

Lack of toothbrush ownership

Minimal fluoride toothpaste use

Limited daily tooth brushing

Frequent consumption of sugary foods and soft drinks (diet)

Non-optimal oral health behaviours and perceptions

Public health facilities

\section{Water fluoridation}

Living conditions

Oral hygiene

Policy making challenges
Recourse generation and allocation

Actions and Interventions
Considerable enthusiasm from the public dental services coupled with adequate funding.

Competing demands on limited budgets/adequate funding Policy and organizational practices

Lack of effective interventions

Effective management systems, including policy and funding responsibility

Integration strategies targeting Aboriginal oral health

\section{Service} providing

challenges
Preventive services

Prevention of risk indicators

Integration of the health care system, with dental services

Lack of comprehensive primary health care models

Diagnoses of dental caries and treatment more often involving oral surgery.

Oral health providers
Limited knowledge of prevention, prohibitive cost of services, intensive marketing of sugary products

Discrimination from health providers 


\begin{tabular}{|c|c|c|}
\hline Main themes & Sub-themes & Final codes \\
\hline & & $\begin{array}{l}\text { Under-acknowledged providers of a range of oral health } \\
\text { services }\end{array}$ \\
\hline & & $\begin{array}{l}\text { Access to reliable manufacturers and suppliers of } \\
\text { equipment contractual agreements }\end{array}$ \\
\hline \multirow{7}{*}{$\begin{array}{l}\text { Access } \\
\text { challenges }\end{array}$} & \multirow[t]{4}{*}{ Financial access } & Difficulty contacting patients \\
\hline & & High rate of failure to attend appointments. \\
\hline & & Attendance patterns. \\
\hline & & $\begin{array}{l}\text { Cost is likely to be a significant barrier (specially in } \\
\text { women). }\end{array}$ \\
\hline & \multirow{3}{*}{$\begin{array}{l}\text { Non-financial } \\
\text { access }\end{array}$} & Consent issues. \\
\hline & & Geographic barriers in accessing oral health services \\
\hline & & $\begin{array}{l}\text { Challenges beyond their control as individuals, including } \\
\text { accessing dental services }\end{array}$ \\
\hline
\end{tabular}

The first theme was identified as 'personal characteristics', with five sub-themes including age, sex, physiological status, psychological status and health literacy status (Table 3). These themes demonstrated how oral health utilization can be influenced and shaped by the personal characteristics of Indigenous Australians. Among the personal characteristics, age is considered as a determinant criterion. Children [30,38], teenagers [12] and elders [21] are supposed as the important age groups. Moreover, women $[12,21,34]$ are more vulnerable to lack of access and utilization to oral health services. Oral health literacy was considered as a risk indicator for poor self-reported oral health outcomes among the indigenous groups [9]. Low levels of oral health literacy may contribute to lack of attention to oral health and late and emergency dental referrals [7] so it will be important for the policy makers to identify more vulnerable groups and help them to become impovered.

The second theme identified was socio-economic status This theme comprised economic status and social and cultural status of the Indigenous Australian population that poses many challenges in the utilization of oral health services. Financial concerns of the families [23,35], transportation problems [8, 29] and living in remote areas [39] were among some of the economic challenges of oral health utilization. Cultural barriers included difficulty in communication [36], language barriers [29] and low selfefficiency [34].

Public health limitations are among other challenges that restrict provision of optimal oral health services among Indigenous Australians. Public health issues in this regard are divided into public health behaviours and public health facilities. The main concept among the Indigenous population according to our findings include beliefs [8] and perceptions [22] that affect oral health-related behaviours. These behaviours range from daily tooth brushing and fluoride toothpaste use to consumption of sugary foods 
and carbonated beverages [25]. Along with public behaviours, public facilities can affect a population's provision to oral health services. Our findings indicated that the communities which have inadequate levels of natural fluoride in the drinking water and high levels of dental caries need greater access to dental public health facilities [27]. Water fluoridation units in the more populated remote Indigenous communities (ie more than 500 people) [27] is a good example of an equitable and cost-saving dental public health intervention.

Policy making challenges including resource allocation and the governments' actions and interventions are considered as the fourth challenges of provision to oral health services (Table 3). Allocating adequate funding to the oral health needs of Indigenous Australians $[27,28]$ was the strongest sub-theme. Including programs that aimed to reduce early childhood caries, increasing services to remote communities, developing the role of Aboriginal health workers, improving oral health literacy, establishing water fluoridation and providing periodontal therapy are some effective interventions that can be compounded by the complex interplay between psychosocial and cultural determinants of both Indigenous Australians and oral health professionals. Public health behaviour and public health facilities were two of the sub-themes in this regard.

Service provision challenges were identified as the fifth theme influencing oral health provision among Indigenous Australians. The emphasis on surgeries as opposed to preventive services was noted [32], together with lack of integration between preventive primary health care systems and dental services [31, 33]. A lack of an existing comprehensive primary health care model [24] can lead to a decrease in provision of oral health services. It was apparent that many Indigenous Australians were unaware of how to make an emergency dental visit and had limited understanding of the prevention of dental disease [35].

Finally, access challenges including financial and non-financial access were among other identified challenges that reduce Indigenous Australian utilization of dental services. Difficulties in contacting patients [36], geographic barriers in accessing oral health services [37], high rates of failure to attend appointments [36] contribute to this. Cost of dental services [26,31] was a significant barrier restricting many Indigenous Australians' access to oral health services.

For a better understanding of the challenges impacting the utilization and provision of oral health services among Indigenous Australians, Fig. 2 clarifies the conceptual map of the present scoping review through a thematic network.

\section{Discussion}

Our findings indicate that many Indigenous Australians encounter challenges and barriers in the utilization and provision of oral health services. Our findings are corroborated by El-Yousfi et al (2019), who identified three categories of determinants as the barrier to oral health services among vulnerable groups. These three determinants include individual, organizational and policy level barriers [40] that can be matched to the present results to some extent. 
The present results have shown that age (children, teenagers, elderly), sex, physiological status, psychological status and oral health literacy status can decrease oral health utilization among Indigenous Australians. Hernández-Palacios et al. (2015) identified that sex, low income and low educational level have a significant influence on the self-perceived oral health status of elderly Mexicans [41]. Lee et al. (2014) described how sex, marital status, income level and educational levels influenced oral health utilization among residents of Ohio in the United States[42].

Similar to our findings regarding socio-economic status, El-Yousfi et al (2019) described how "cultural differences in oral healthcare seeking behaviour" was one cultural barrier and the "limited ability to express need for dental treatment" as the main economic challenge [40]. Watt et al. (2019) provided evidence that Indigenous populations at a global level are vulnerable adult population groups in utilizing oral health services. They described how a population`s social class, income, education, psychosocial factors and social relationships are considered as the intermediate determinants that affect the utilization of oral health services [43]. Biordi et al. (2015) also emphasized how barriers to utilize oral healthcare remain significant in low-income children [44]. In that the Indigenous Australian context, access to social supportive packages may help overcome the economic barriers of oral healthcare utilization.

Watt et al. described how resources were an enabler to access oral health services [43], with Dahlan et al. (2019) also referring to social support as a determinant of access to oral healthcare among immigrants and ethnic minorities [45]. A report for the Waterloo Region Oral Health Coalition in Canada (2017) emphasized how cost is a huge barrier to accessing oral health care along with the clients` oral health literacy, fear and anxiety [46].

tour findings identify public health challenges as one of the barriers to provision of oral health services among Indigenous Australians. This is supported by Levesque et al. (2013) who emphasized that ability to perceive health care needs containing health beliefs, expectations and literacy can lead to access to health care services [47]. Similarly, Göstemeyer et al. (2019) indicated that lack of oral health knowledge, beliefs and behaviors can act as barriers to access oral healthcare. They described how oral hygiene of patients was a determinant barrier in provision of oral and dental treatment [48]. Regular dental visits together with access to water fluoridation and other public health interventions are both beneficial and cost-effective in this regard.

Policy makers have a multi-disciplinary role in improving provision of the oral health services. They can set the agenda to include wider discussion around provision of oral services, advocate stakeholders and allocate more resources to this area. Designing and managing of effective population-level interventions can be considered by national and local policy makers to support the oral health needs of all socially excluded populations, Indigenous Australians among them.

Balasubramanian et al (2019) have shown that some problems in dental service utilisation arise from narrow approaches to dental education and imbalances among the skills, jobs and competencies of oral service providers. Macro challenges of the health care system exist, including imbalanced geographic 
distribution and an increasing divide between the 'professions' interests, and needs of the population [49]. These problems can restrict both access and utilisation of oral health services.

\section{Limitations}

Study limitations include: a quantitative approach for analysing oral health indicators among Indigenous Australians would have provided useful information in conjunction with the scoping review. That would have enabled the present results to be triangulated with the face to face interviews from Indigenous participants to better explore other probable reasons of challenges in the utilization of oral health services.

\section{Conclusion}

Our findings indicate that the utilization and provision of oral health services for Indigenous Australians can be restricted via personal and socioeconomic factors. Challenges relating to policy making, service providing, public health and access can affect their utilization and provision. Policy makers in a national and local level can plan to decrease these limitations although most need long term, multidisciplinary interventions.

\section{Delecrations}

\section{Ethics approval and consent to participate}

Not applicable.

\section{Consent for publication}

Not applicable.

\section{Availability of data and material}

All data generated or analysed during this study are included in this published article and the appendix.

\section{Competing interests}

The authors declare that they have no competing interests.

\section{Funding}

There was no funding.

\section{Authors' contributions}


PB is designed the study and finalize the dasta analysis. YS, AG and FO are collected the data and extracted the data. LJ is technically edited and finalized the article.

\section{Acknowledgements}

Not applicable.

\section{References}

1. Health Do, Human Services W, DC., and People H, Healthy people 2010: Understanding and improving health. 2000: US Department of Health and Human Services.

2. Mohammadpour $\mathrm{M}$, et al. Oral health policymaking challenges in Iran: a qualitative approach. BMC Oral Health. 2020;20(1):158.

3. Petersen PE, et al. The global burden of oral diseases and risks to oral health. Bull World Health Organ. 2005;83:661-9.

4. Dahlan $\mathrm{R}$, et al., Impact of social support on oral health among immigrants and ethnic minorities: $A$ systematic review. PloS one, 2019. 14(6).

5. Tiwari T, et al. Reducing indigenous oral health inequalities: a review from 5 nations. Journal of dental research. 2018;97(8):869-77.

6. Fredericks B, et al., Aboriginal and Torres Strait Islander health. Professional Nursing and Midwifery Practice [Custom Edition for Monash University], 2020: p. 264.

7. Irving $M$, et al. Client perspectives on an Aboriginal community led oral health service in rural Australia. Australian journal of rural health. 2017;25(3):163-8.

8. Tynan A, et al. Factors influencing the perceived importance of oral health within a rural Aboriginal and Torres Strait Islander community in Australia. BMC Public Health. 2020;20:1-9.

9. Parker EJ, Jamieson LM. Associations between indigenous Australian oral health literacy and selfreported oral health outcomes. BMC Oral health. 2010;10(1):3.

10. Durey A, Bessarab D, Slack-Smith L. The mouth as a site of structural inequalities; the experience of Aboriginal Australians. Community Dent Health. 2016;33(2):161-3.

11. Durey A, et al. Aboriginal Health Worker perceptions of oral health: a qualitative study in Perth, Western Australia. Int J Equity Health. 2016;15(1):4.

12. Butten $\mathrm{K}$, et al. Yarning about oral health: perceptions of urban Australian Aboriginal and Torres Strait Islander women. BMC Oral Health. 2020;20(1):35.

13. Donato R, Segal L. Does Australia have the appropriate health reform agenda to close the gap in Indigenous health? Aust Health Rev. 2013;37(2):232-8.

14. Spencer AJ, Harford JE, Oral health of Australians: National planning for oral health improvement. 2001: South Australian Department of Human Services. 
15. National. Advisory Committee on Oral Health.Healthy Mouths, Healthy Lives: Australia's national oral health plan 2004-2013. 2004.

16. Nguyen H. Patient centred care: cultural safety in indigenous health. Aus Fam Physician. 2008;37(12):990.

17. Dyson $K$, Kruger $E$, Tennant M. A decade of experience evolving visiting dental services in partnership with rural remote Aboriginal communities. Australian dental journal. 2014;59(2):187-92.

18. Arksey H, O'Malley L. Scoping studies: towards a methodological framework. Int J Soc Res Methodol. 2005;8(1):19-32.

19. Munn Z, Peters MD, Stern C, Tufanaru C, McArthur A. \& Aromataris, E. (2018)..

20. Systematic review or scoping review? Guidance for authors when choosing between a.

21. systematic or scoping review approach. BMC Medical Research Methodology, 18 (1).

22. $143 .</$ number\&gt.

23. Thomas, J., \& Harden, A. (2008). Methods for the thematic synthesis of qualitative.

24. research in systematic reviews. BMC Medical Research Methodology, 8(1), 45. .

25. Amarasena N, et al. (2015). "Psychological distress and self-rated oral health among a convenience sample of Indigenous Australians." Journal of Public Health Dentistry 75(2): 126-133..

26. Amarasena $\mathrm{N}$, et al. (2014). "Oral health behaviours and perceptions reported by Indigenous Australians living in Darwin, Northern Territory." Community Dent Health 31(1): 57-61..

27. Butten K, et al. (2019). "Impact of oral health on Australian urban Aboriginal and Torres Strait Islander families: a qualitative study." International Journal for Equity in Health $18 .$.

28. Campbell MA, et al. (2015). "The oral health care experiences of NSW Aboriginal Community Controlled Health Services." Australian and New Zealand Journal of Public Health 39(1): 21-25..

29. Dimitropoulos Y, et al. (2018). "Aboriginal communities in rural New South Wales, Australia to determine the oral health needs of their children develop a community-owned oral health promotion program." Rural and Remote Health 18(2)..

30. Durey A, et al. (2017). "Oral Health in Young Australian Aboriginal Children: Qualitative Research on Parents' Perspectives." JDR Clin Trans Res 2(1): 38-47..

31. Ehsani JP and Bailie R (2007). "Feasibility and costs of water fluoridation in remote Australian Aboriginal communities." BMC Public Health 7: 100..

32. Green J. and A. Blinkhorn (2010). "How can inequalities in the oral health of Australian Aboriginal people be addressed?" International Journal of Health Promotion and Education 48(4): 107-110..

33. Jones KBAMPHP, et al. (2016). "Factors Relating to Access to Dental Care for Indigenous South Australians." Journal of Health Care for the Poor and Underserved 27(1): 148-160..

34. Patel J, et al. (2017). "Oral health interventions in Australian Aboriginal communities: a review of the literature." Australian Dental Journal 62(3): 283-294..

35. Schluter PJ, et al. (2017). "Aboriginal and Torres Strait Islander oral health and its impact among adults: A cross-sectional study." Australian Dental Journal 62(1): 84-94.. 
36. Slater PJ. (2001). "Patterns of access to public oral health care in Queensland by gender, indigenous status and rurality." Australian Dental Journal 46(2): 122-133..

37. Smith K, et al. (2007). "Oral health in rural and remote Western Australian Indigenous communities: A two-year retrospective analysis of 999 people." International Dental Journal 57(2): 93-99..

38. Jamieson LM, Parker EJ, Roberts-Thomson KF, Lawrence HP, Broughton J. Self-efficacy and selfrated oral health among pregnant aboriginal Australian women. BMC Oral Health. 2014; 14:29. Published 2014 Apr 2.

39. Jones K, Parker EJ, Jamieson LM. Access, literacy and behavioural correlates of poor self-rated oral health amongst an indigenous south Australian population. Community Dent Health. 2014;31(3):167-71.

40. Parker EJ, Misan G, Shearer M, et al. Planning, implementing, and evaluating a program to address the oral health needs of aboriginal children in port augusta, australia. Int $\mathrm{J}$ Pediatr. 2012;2012:496236.

41. Williams S, Jamieson L, MacRae A, Gray C. (2011) Review of Indigenous oral health. Australian Indigenous HealthBulletin 11(2).

42. Jamieson LM, Armfield JM, Roberts-Thomson KF. The role of location in indigenous and nonindigenous child oral health. J Public Health Dent. 2006;66(2):123-30.

43. Kruger E, Smith K, Atkinson D, Tennant M. The oral health status and treatment needs of Indigenous adults in the Kimberley region of Western Australia. Aust J Rural Health. 2008;16(5):283-9.

44. El-Yousfi S, Jones $\mathrm{K}$, White S, Marshman Z. A rapid review of barriers to oral healthcare for vulnerable people. Br Dent J. 2019;227(2):143-51.

45. Hernández-Palacios RD, Ramírez-Amador V, Jarillo-Soto EC, Irigoyen-Camacho ME, Mendoza-Núñez VM. Relationship between gender, income and education and self-perceived oral health among elderly Mexicans. An exploratory study. Ciência Saúde Coletiva. 2015;20(4):997-1004.

46. Lee W, Kim SJ, Albert JM, Nelson S. Community factors predicting dental care utilization among older adults. J Am Dent Assoc. 2014;145(2):150-8.

47. Watt RG, Venturelli R, Daly B. Understanding and tackling oral health inequalities in vulnerable adult populations: from the margins to the mainstream. Br Dent J. 2019;227(1):49-54.

48. Biordi DL, et al. Improving Access and Provision of Preventive Oral Health Care for Very Young, Poor, and Low-Income Children Through a New Interdisciplinary Partnership. American Journal of Public Health Supplement 2, 2015, Vol 105, No. S2.

49. Dahlan R, Ghazal E, Saltaji H, Salami B, Amin M. Impact of social support on oral health among immigrants and ethnic minorities: A systematic review. PLoS One. 2019;14(6):e0218678.

50. Horst K. Barriers to Accessing Oral Health Care. A report for the Waterloo Region Oral Health Coalition. April 2017.

51. Levesque JF, Harris MF, Russell G. Patient-centred access to health care: conceptualising access at the interface of health systems and populations. Int J Equity Health. 2013;12:18. 
52. Göstemeyer G, Baker SR, Schwendicke F. Barriers and facilitators for provision of oral health care in dependent older people: a systematic review. Clin Oral Investig. 2019;23(3):979-93.

53. Balasubramanian M, Brennan DS, Short SD, Gallagher JE. A strife of interests: A qualitative study on the challenges facing oral health workforce policy and planning. Health Policy. 2019 Nov;123(11):1068-75.

Figures

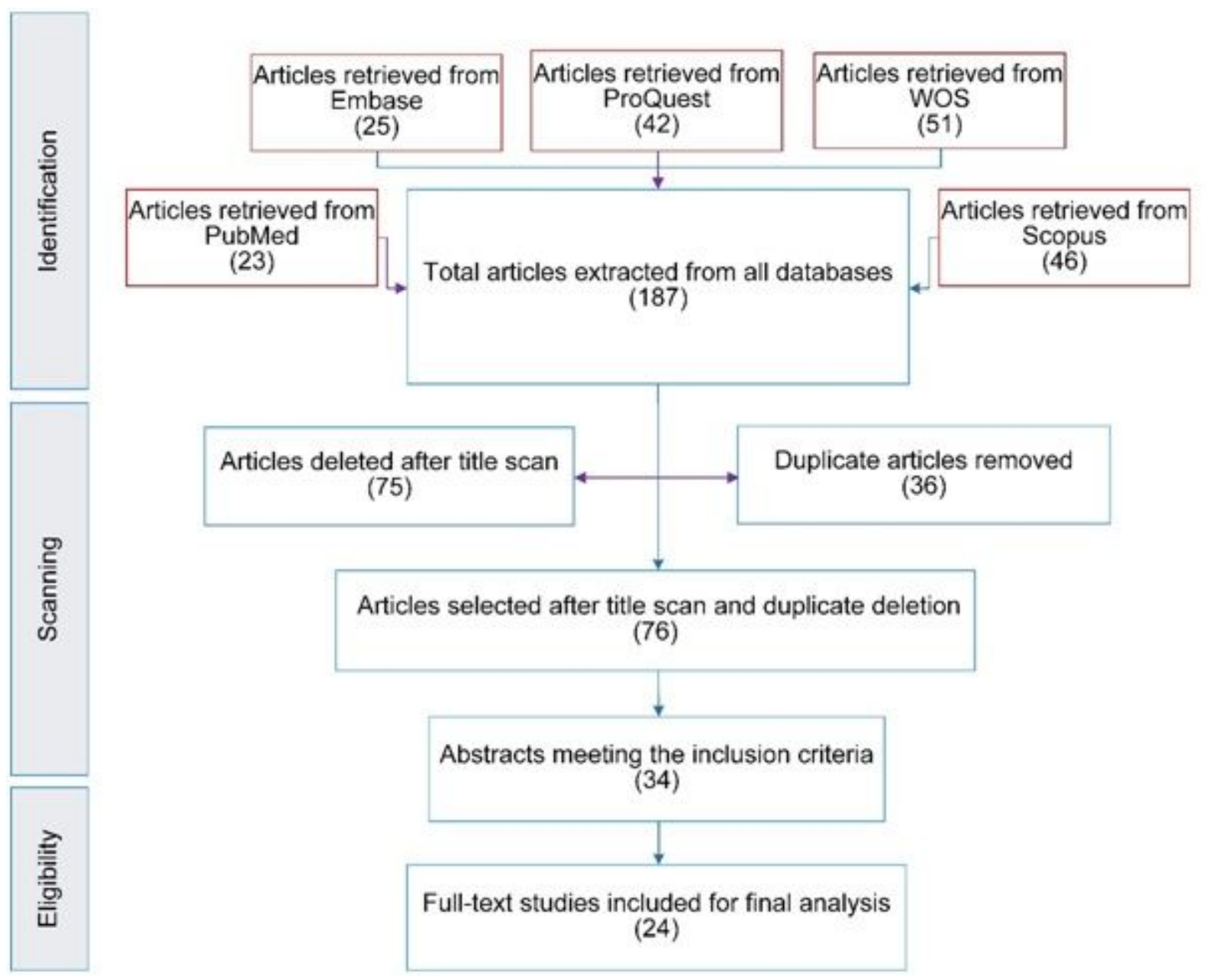

Figure 1

The PRISMA flowchart of the scoping review 


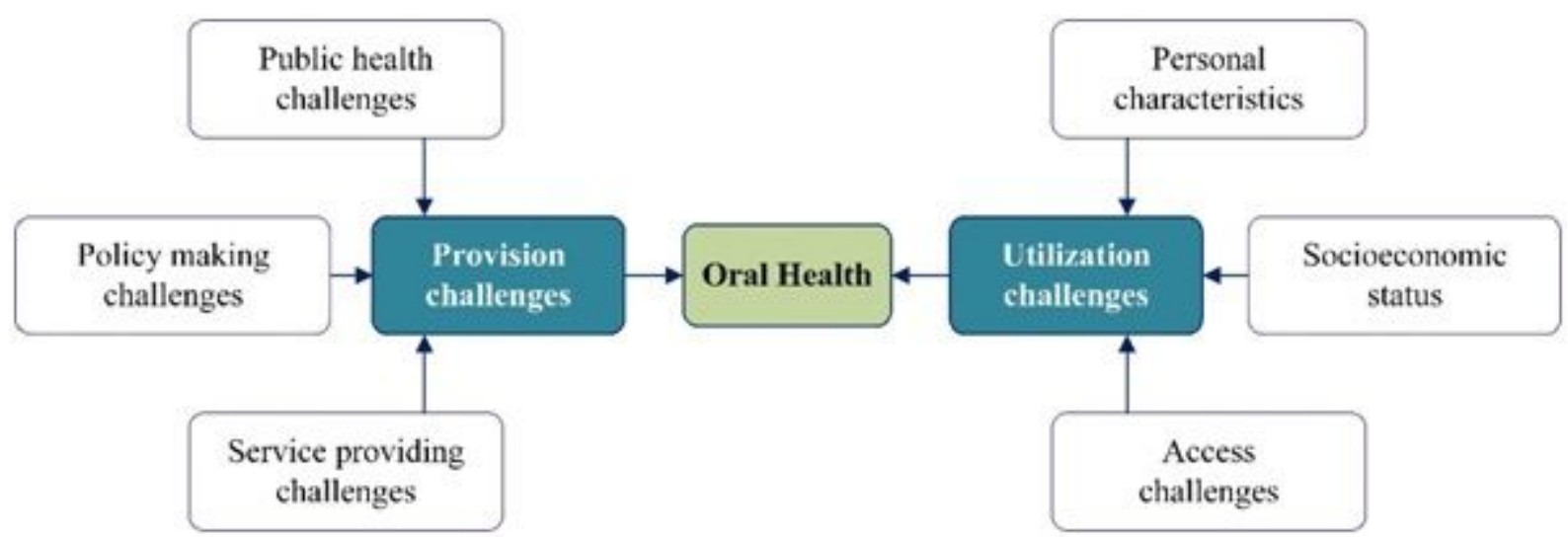

\section{Figure 2}

the conceptual framework and the thematic mapof the scoping review

\section{Supplementary Files}

This is a list of supplementary files associated with this preprint. Click to download.

- Appendix.docx

- Appendix.docx 